\title{
AMPLE: A Novel Incentive Approach to Adaptive-Width Channel Allocation in Multi-hop, Non-cooperative Wireless Networks ${ }^{\star}$
}

\author{
Chunyang $\mathrm{Wu}^{1}$, Fan $\mathrm{Wu}^{1, \star \star}$, Guihai Chen ${ }^{1}$, and Bo Sheng ${ }^{2}$ \\ 1 Shanghai Key Laboratory of Scalable Computing and Systems, \\ Department of Computer Science and Engineering, \\ Shanghai Jiao Tong University \\ 2 Department of Computer Science, University of Massachusetts Boston \\ chunyang506@sjtu.edu.cn, \{fwu,gchen\}@cs.sjtu.edu.cn, \\ shengbo@cs.umb. edu
}

\begin{abstract}
Due to the limitation of radio spectrum resource and fast deployment of wireless devices, careful channel allocation is of great importance for mitigating the performance degradation caused by interference among different users in wireless networks. Most of existing work focused on fixed-width channel allocation. However, latest researches have demonstrated that it is possible to combine contiguous channels for better utilizing the available channels. In this paper, we study the problem of adaptive-width channel allocation in multi-hop, non-cooperative wireless networks from a game-theoretic point of view. We first present a strategic game model for this problem and demonstrate the existence of Nash Equilibrium (NE) in an anarchical scenario. Since a NE is not an ideal solution, we then propose AMPLE, a novel incentive approach to guarantee the system performance at high level. Since the problem of channel allocation in multiple collision domains is NP-complete, we first present an approximate algorithm that gives an allocation with good system performance. We then design a charging scheme that guarantees the system to converge to a Dominant Strategy Equilibrium (DSE), in which it is to the best interest of each node to follow the computed channel allocation, regardless how the others behave. Numerical results verify that AMPLE does prevent nodes' misbehavior, and achieves much higher average system throughputs than anarchical NEs.
\end{abstract}

\section{Introduction}

Due to historical reasons, radio spectrum is manually divided into communication channels, and each channel is assigned to a specific application in a geographic area. For instance, the commonly used IEEE 802.11 standard specifies several orthogonal channels (e.g., 3 in IEEE 802.11b/g and 12 in IEEE 802.11a). Such static channelization prevents the limited radio spectrum from being used efficiently [11,15, 18]. Furthermore,

\footnotetext{
* This work was supported in part by China NSF grant 61170236 and 61133006 . The opinions, findings, conclusions, and recommendations expressed in this paper are those of the authors and do not necessarily reflect the views of the funding agencies or the government.

${ }^{\star \star}$ Corresponding author.
}

X. Wang et al. (Eds.): WASA 2012, LNCS 7405, pp. 43-59, 2012.

(C) Springer-Verlag Berlin Heidelberg 2012 
the United States has completed its transition to fully digital television broadcasting on June 12, 2009, and opened up unlicensed use of TV whitespaces that span 100-250 $\mathrm{MHz}$ of spectrum [1]. This raises the need for dynamic spectrum allocation.

Ranveer Chandra et al. [6] proposed that the width of IEEE 802.11-based communication channels can be changed adaptively in software by using commodity Wi-Fi hardware. For example, two contiguous $20 \mathrm{MHz}$ channels can be combined into a 40 $\mathrm{MHz}$ channel to provide higher bit-rate. Furthermore, the emergence of cognitive radio makes it more convenient to adaptively utilize available radio spectrum. Although the problem of channel allocation has been extensively studied in the literature, the feature of adaptive-width channel has not been fully considered [22].

Since nodes equipped with cognitive radio can easily adapt themselves to operate in any part of radio spectrum spaces, we can no longer assume that the nodes in the network would follow the prescribed spectrum allocation protocol faithfully. The most rational strategy for an individual node is to tune its wireless interface to the available spectrum (channel), in which it can get the best payoff. However, such selfish behavior may degrade the networks performance, due to inefficient channel allocation. In this paper, we consider the problem of adaptive-width channel allocation in non-cooperative wireless networks, where the participating nodes are always selfish and pursue their own objectives. Wu et al. [22] presented an incentive scheme to guarantee the system to converge to a state, in which system-wide throughput is optimized. However, their work only applies to a single-hop network, wherein all transmissions on the same channel will collide with each other. This limits the practical usage of the proposed incentive scheme, because spatially well separated transmissions can work on the same channel simultaneously. For example, in a large building, two well separated access points can serve wireless users using the same channel. Therefore, we will study the problem of adaptive-width channel allocation in multi-hop, non-cooperative wireless networks, and propose our strong and practical solution.

To understand the impact of participating nodes' selfish behavior, we first model the problem of adaptive-width channel allocation as a strategic game, and study the Nash equilibrium (NE) the system converge to, when there is no exogenous factor to influence the nodes' behavior. We introduce a simple algorithm to simulate selfish nodes' behaviors, and to compute a NE the system may converge to. Although the algorithm cannot enumerate all the possible NEs, its outputs provide us the following understanding of the NE:

1. NE is not a strong equilibrium for all the players to comply with. In a NE scenario, only under the assumption that all other players kept their equilibrium strategies would a player of the game have incentives to keep its equilibrium strategy. Thus NE does not provide strong incentives for the game player.

2. NE is usually not globally efficient, which means that the maximized system-wide performance is not always achieved. So, even if the system converged to one of the NEs, some player might benefit at the cost of system-wide performance degradation.

3. Although our algorithm finishes in $O(n c)$ steps, where $n$ is the number of nodes in the network and $c$ is the number of available channels, the convergence may take extremely long time in practice. 
Therefore, NE is not an ideal solution to the problem of adaptive-width channel allocation, and we need to seek stronger solutions that can guarantee the system performance at high level.

To achieve strong incentives and to maintain high system performance, we propose an incentive scheme, namely AMPLE, that can guarantee the system converging to a Dominant Strategy Equilibrium (DSE), a novel incentive approach to Adaptive-width channel allocation in Multi-hop, non-cooPerative wireLess nEtworks. In game theory, DSE is a solution much stronger than NE. For each node, instead of going through a complicated decision process, simply picking its corresponding strategy in the DSE is the best strategy, regardless of the others' strategies. In the meanwhile, the system-wide performance achieved in the DSE is guaranteed to be high.

The major contributions of this paper are as follows:

1. First, to our knowledge, we are the first to study the problem of adaptive-width channel allocation in multi-hop, non-cooperative wireless networks. Our solution is strong and practical.

2. Second, we present an algorithm to simulate the selfish behavior of the nodes. The results of the algorithm show that there exist multiple NEs the system may converge to. More importantly, NE is not a perfect solution concept to the problem studied in this paper.

3. Third, we propose an incentive scheme that can guarantee the convergence of the system to a Dominant Strategy Equilibrium (DSE), in which the system-wide performance achieved in the DSE is guaranteed to be high.

The rest of the paper is organized as follows. In Section 2, we present our system model, game model and some necessary concepts. In Section 3, we show the existence of NE in anarchy. In Section 4, we propose AMPLE, as our solution to the problem. In Section5 we report the evaluation results. In Section 6 , we give a brief review of the related work. Finally, in Section 7, we conclude this paper and put forward potential future work.

\section{Preliminaries}

\subsection{System Model}

In this paper we consider a static wireless network with some access points. Each access point is equipped with a radio interface and can provide data service within its coverage area. Define $N \triangleq\{1,2,3, \ldots, n\}$. Figure 1(a) illustrates a proper example. There are three access points (AC) $A, B$ and $C$. The dotted circles are the coverage areas of those ACs. In this scenario, A conflicts with $\mathrm{C}$ while not with $\mathrm{B}$.

Given a set of channels donated by $C \triangleq\{1,2, \ldots, c\}$, we assume that the channels are contiguous, orthogonal (non-interfering), and homogenous. Since the access points need the channels to provide services to their customers, we want to efficiently allocate the channels to the access points. Due to service quality requirement, we require that the access points do not have any channel conflict with each other. By treating each access point as a node in the graph, we set up a conflict graph $G \triangleq(N, E)$, where $E$ represents the conflict edge set, $e=(i, j) \in E$ means that $j$ and $i$ conflict with each other. 


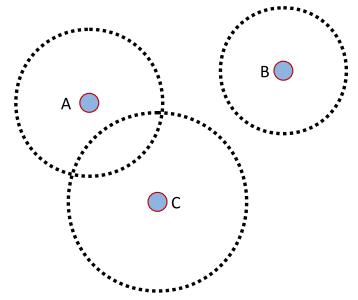

(a) An example showing confliction between access points.

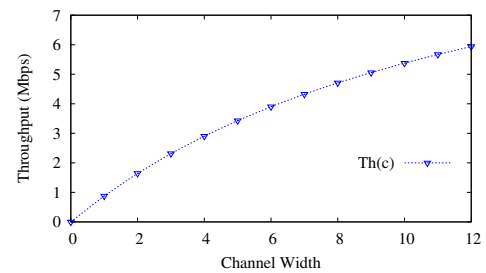

(b) Properties of the effective aggregate throughput $T h(c)$.

Fig. 1.

For any access point $i \in N$, We denote its allocated channel set by $\mathbb{C}(i)$.

We require that the channels allocated to an access point must be contiguous. An AC can obtain a higher throughput by combining contiguous channels into a wider on Let $T h(c)$ represent the effective aggregated throughput of a channel with the bandwidth of $c$ original channels. As shown in [4], $T h(c)$ is a concave non-decreasing function of $c$. Figure 1(b) illustrates these properties of $T h(c)$.

For a particular AC, it is able to combine contiguous channels, which are not conflicting with its neighbors. Thus its throughput is that of the combined channel.

Definition 1 (Longest Contiguous Segment (LCS)). Given an integer set $A$, a contiguous segment is subset that requires the elements are contiguous. We define $\operatorname{LCS}(A)$ as the longest contiguous segment in A.

Based on this definition, we formulate the throughput of $\mathrm{AC} i$ as

$$
T_{i}(s)=T h(|L C S(\mathbb{C}[i])|) .
$$

\subsection{Game Model}

We model the adaptive-width channel allocation as a strategic game. In this game, we treat the access points as players. We assume the players are rational and do not collude or cooperate with each other. The strategy of player $i \in N$ is its allocated channel set:

$$
s_{i} \triangleq \mathbb{C}(i) .
$$

In the rest of this paper, we use $s_{i}$ and $\mathbb{C}(i)$ interchangeably.

The strategy profile $s$ is a vector composed of all the players' strategies,

$$
s \triangleq\left(s_{1}, s_{2}, \ldots, s_{n}\right)^{T} .
$$

Conventionally, $s_{-i}$ represents the strategy profile of the other players except player $i$.

For a strategy profile $s$, let's denote the throughput of player $i$ by $T_{i}(s)$. As mentioned previously, the allocated channels of one player should be contiguous. If it selects some separated channels that can not be combined, it can not fully utilize them. 
We then define a player's utility. As in the literature (e.g. [8, 20, 26, 28]), we assume that there exists some kind of virtual currency in the system. In this paper, we define the utility of player $i$ as

$$
u_{i}(s) \triangleq \alpha T_{i}(s)-\mathcal{P}_{i}(s)
$$

where $\alpha$ is a coefficient and $\mathcal{P}_{i}(s)$ represents the charge to player $i$ for using channels. Since a player cannot guarantee the quality of the service provided to its customers, we let $u_{i}(s) \triangleq-\mathcal{P}_{i}(s)$, when the player collide with one of its conflicting neighbors.

We then review some solution concepts from game theory used in this paper.

Definition 2 (Nash Equilibrium [17]). A strategy profile $s^{*}$ is a Nash Equilibrium of a strategic game, if for any player $i \in N$ and for any strategy $s_{i} \neq s_{i}^{*}$,

$$
u_{i}\left(s_{i}^{*}, s_{-i}^{*}\right) \geq u_{i}\left(s_{i}, s_{-i}^{*}\right)
$$

Definition 3 (Dominant Strategy Equilibrium [10,17]). A strategy profile $s^{*}$ is a dominant strategy equilibrium of a strategy game, if for any player $i \in N$, any strategy $s \neq s^{*}$ and any strategy profile of the other players $s_{-i}$,

$$
u_{i}\left(s_{i}^{*}, s_{-i}\right) \geq u_{i}\left(s_{i}, s_{-i}\right) .
$$

\section{Anarchical Nash Equilibrium}

In this section, we show the existence of Nash Equilibrium (NE) in anarchy, when there is no external factor to influence the players' behaviors. Each player's objective is to maximize its own throughput and hence the utility of the player is

$$
u_{i}(s)=\alpha T_{i}(s)
$$

if it does not collide with its conflicting neighbors; otherwise

$$
u_{i}(s)=0 .
$$

\subsection{Computing NE}

The pseudo-code for computing a NE is showed in Algorithm 1 We first successively allocate each node a random available channel (Line 1,7). We denote the set of adjacent nodes to a node as

$$
\operatorname{Adj}[i] \triangleq\{j \mid(i, j) \in E\} .
$$

Then, we check each node and update its allocated channel(s) if it can get its throughput improved with the new allocation (Line 8, 12). We repeat the above process until no node can improve its throughput by jumping to another set of contiguous channels. We denote the set of adjacent nodes to a node as

$$
A d j[i] \triangleq\{j \mid(i, j) \in E\} .
$$




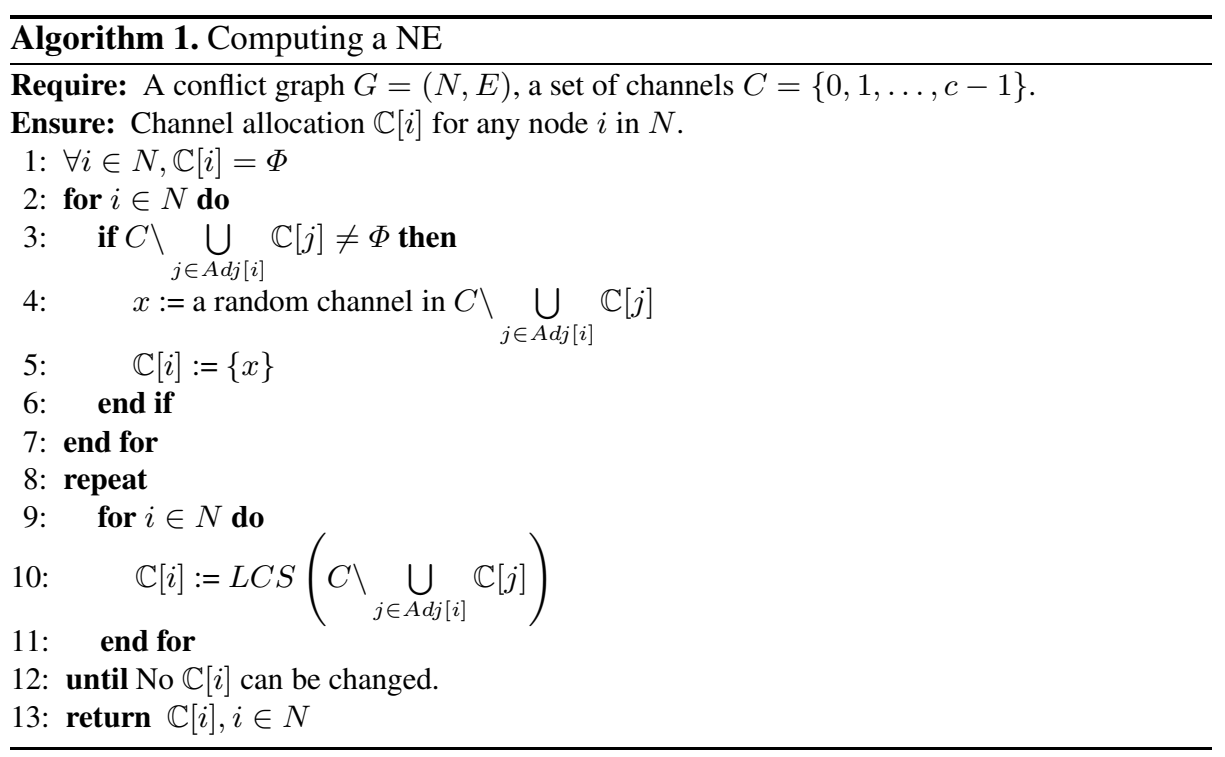

\subsection{Analysis}

We prove the channel allocation strategy profile $s^{*}$ determined by $\mathbb{C}[i], i \in N$, which is computed by Algorithm 1, is a NE.

Theorem 1. The channel allocation strategy profile $s^{\star}$ computed by Algorithm 1 is a NE.

Proof. Since conflicting access points can not share any channel, the throughput of each conflicting ones will be zero if they share some channels. Hence for any strategy profile $s$ and any player $i$ in $N, u_{i}(s)=0$ if $\mathbb{C}[i] \cap \bigcup_{j \in A d j[j]} \mathbb{C}[j] \neq \Phi$.

If for a node $i$, we choose another $\mathbb{C}^{\prime}[i]$. Denote this new strategy by $s_{i}$. Let $s=$ $\left(s_{i}, s_{-i}^{*}\right)$. We distinguish two cases:

1. $\mathbb{C}^{\prime}[i] \cap \bigcup \mathbb{C}[j] \neq \Phi$. This happens when Player $i$ collides with its neighbors. $j \in \operatorname{Adj}[j]$

In this case, it is not able to utilize the channel, so $u_{i}(s)=0 \leq u_{i}\left(s^{*}\right)$.

2. $\mathbb{C}^{\prime}[i] \cap \bigcup \mathbb{C}[j]=\Phi$. This means that Player $i$ combines another set of channels. $j \in \operatorname{Adj}[j]$

Let $D=C \backslash \bigcup_{j \in A d j[i]} \mathbb{C}[j]$. On one hand, Algorithm 11 ensures $\mathbb{C}[i]=\operatorname{LCS}(D)$.

On the other hand, $\mathbb{C}^{\prime}[i] \subseteq D$. So, $\left|L C S\left(\mathbb{C}^{\prime}[i]\right)\right| \leq|L C S(D)|=|\mathbb{C}[i]|$. Therefore $u_{i}(s) \leq u_{i}\left(s^{*}\right)$.

We can conclude that for any player $i$ and for any strategy profile $s=\left(s_{i}, s_{-i}^{*}\right)$,

$$
u_{i}(s) \leq u_{i}\left(s^{*}\right) .
$$

The result computed by Algorithm 1 is a NE. 


\section{Design of AMPLE}

$\mathrm{NE}$ is not an ideal solution concept. As we have mentioned, given a particular network topology, there may exist many NEs and the global performance might vary in a wide range in different NEs. Figure 2 illustrates an example of comparing two NEs.
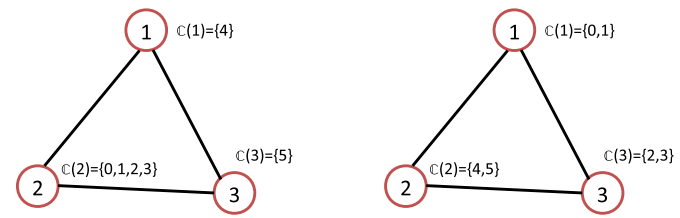

Fig. 2. An example of comparing two NEs. The right one gives a better global throughput than the left one, when there are 6 channels and 3 access points.

In this section, to cope with the weakness of $\mathrm{NE}$, we propose our carefully designed incentive scheme AMPLE. AMPLE consists of two components. One is an approximate algorithm that gives an allocation with good system performance. To stimulate the access points to follow this allocation, the other part is a charging scheme to guarantee that following the computed channel allocation is the dominant strategy of each access point. Thus AMPLE guarantees the system to converge to a Dominant Strategy Equilibrium (DSE).

\subsection{Channel Allocation}

We now introduce our channel allocation algorithm. Our procedure can be divided into two phases. The first phase (Algorithm 2) converts the original conflict graph $G \triangleq(N, E)$ into a directed acyclic graph $\tilde{G} \triangleq(N, \tilde{E})$. Based on $\tilde{G}$, the second phase (Algorithm 3) specifies the channels allocated to each node.

Constructing $\tilde{G}$. In this phase, we convert $G$ into a directed acyclic graph $\tilde{G}=(N, \tilde{E})$. The pseudo-code is listed in Algorithm 2 .

Based on nodes' degrees, we divide $N$ into several independent sets and give each node an order to represent which set it is in (Line 109). Let $\operatorname{Ord}(i)$ represent the order of node $i$. The loop iteratively finds a node $i$ of maximal degree, remove $i$ and its edges. If $i$ 's degree is equal to that of last node $j$, indicating that $i$ and $j$ do not share an edge (or $j$ is not with maximal degree), then let $\operatorname{Ord}(i):=\operatorname{Ord}(j)$. Otherwise, let $\operatorname{Ord}(i):=\operatorname{Ord}(j)+1$. We record the maximal order as $\mathscr{O}$. Next we construct the directed acyclic graph $\tilde{G}=(N, \tilde{E})$ based on the nodes' orders (Line 10 17). For any edge $(i, j)$ in $E$, if $\operatorname{Ord}(i)<\operatorname{Ord}(j)$ then we add $\langle j, i\rangle$ to $\tilde{E}$; otherwise we add $\langle i, j\rangle$ to $\tilde{E}$. 


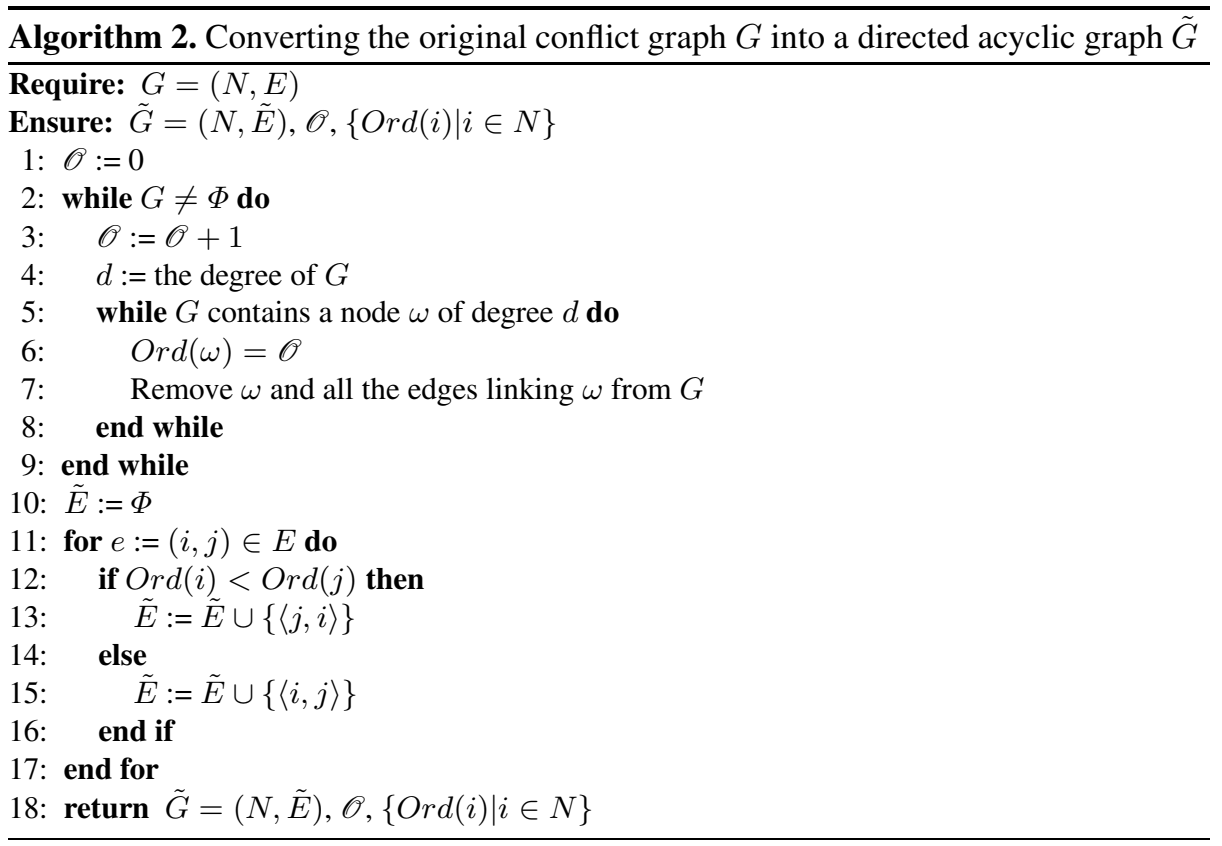

Allocating Channels. We then show the details in Algorithm 3, which computes the channel allocation.

For any node $i \in N$, we define

$$
\operatorname{prev}(i) \triangleq\{s \in \tilde{N} \mid\langle s, i\rangle \in \tilde{E}\} .
$$

We first initialize $\mathcal{L}(i)$ (Line 199). Here $\mathcal{L}(i)$ is a label which we subsequently use to identify its channels. We give each node a label as the minimal element in $N \backslash \bigcup_{j \in \text { prev }(i)}\{\mathcal{L}(j)\}$. In the loop, we record the maximal $\mathcal{L}(i)$ as $\mathscr{L}$ (Line 6).

In line 10, 18, we give each node an original set of channels based on $\mathcal{L}(i)$. If $|C|<$ $\mathscr{L}+1$, we give each node that satisfies $\mathcal{L}(i)<|C|$ one channel (Line 12). Otherwise we give each node $\lfloor\mathcal{L}(i)|C| /(\mathscr{L}+1)\rfloor$ channels (Line 16).

Similar to Algorithm 1 , we then amend the allocation and try to broaden the nodes' channels (Line 19,23).

We show an example of the allocation algorithm in Figure 3

Time Complexity. For Algorithm 2 , constructing $\tilde{G}$ takes $O(|N||E|)$ time. In Algorithm 3, calculating $\mathcal{L}(i)$ takes $O(|N|)$ time; computing the original channels takes $O(|N|)$ time; in the worst situation, amending the channels takes $O(|N||C|)$ time. In sum the upper bound of the time complexity is $O(|N||E|+|N||C|)$.

\subsection{Design of Charging Scheme}

As we have mentioned, NE does not provide a perfect solution to the problem of adaptive width channel allocation. In this section, we propose a charging scheme to make the 


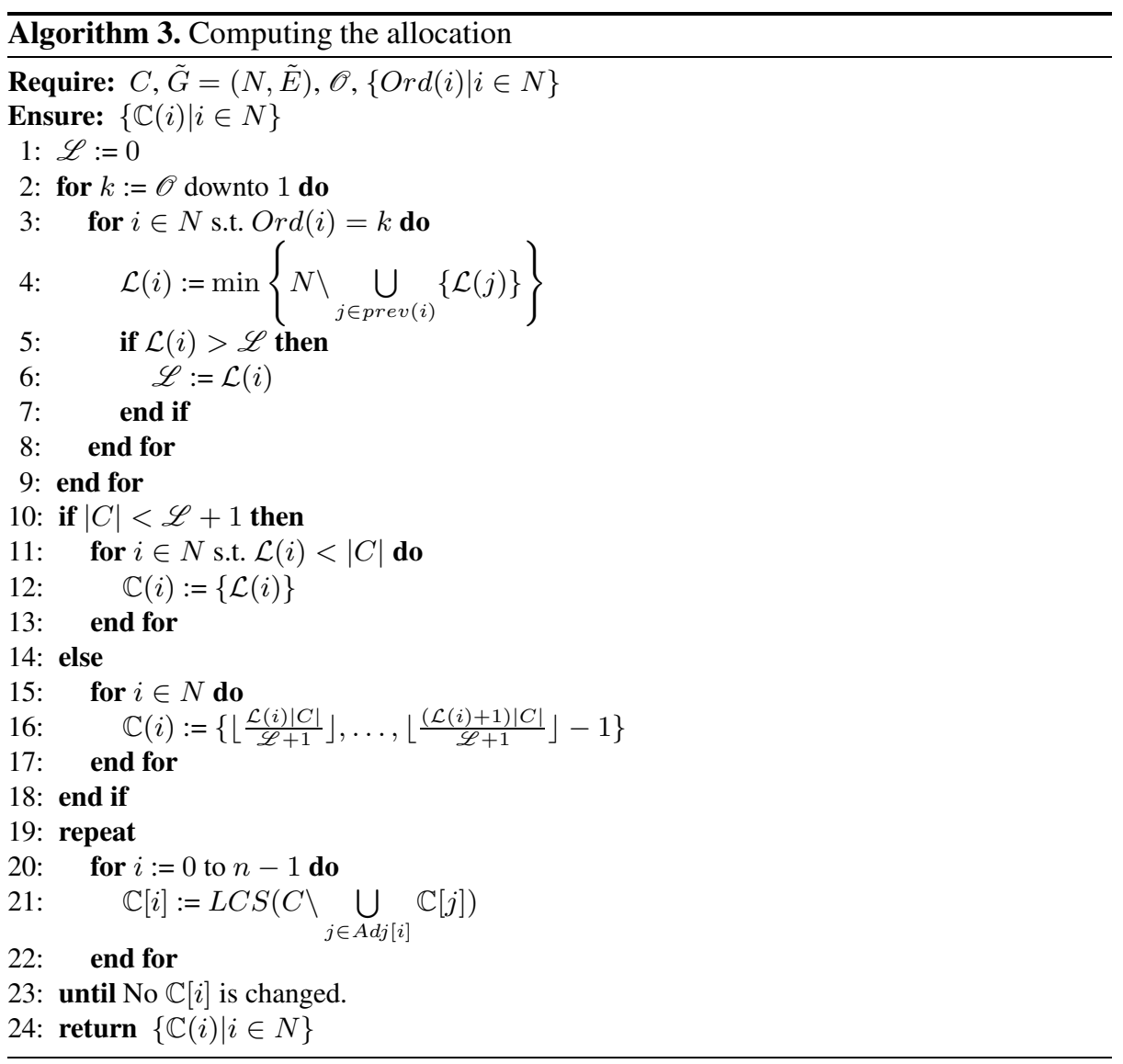

system converge to an equilibrium state, called Dominant Strategy Equilibrium (DSE). This scheme is proposed for two objectives:

1. The charging scheme surely triggers the system's convergence to a DSE, which is a stable state that all the players follows the allocation proposed computed by AMPLE.

2. The charge should be rational and as little as possible. This is because a big or even tremendous charge or punishment would lead no player join the scheme. Exactly as a forfeit of one million dollars for a small mistake like not handling the homework on time is not adopted in real life. An unreasonable charge scheme would strip the significance of the scheme.

Donate the strategy profile determined in Algorithm 3 by $s^{*}$. We next introduce a charging formula, which is a virtual currency [8, 20, 26- 28] to incentive the players' behaviors. 


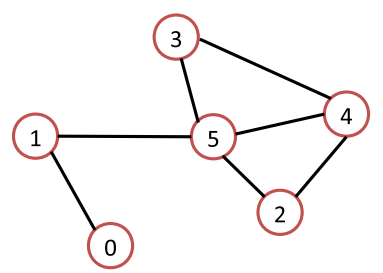

(a) The original conflict graph (b) Divide the nodes into 4 (c) Construct $\tilde{G}=(N, \tilde{E})$. $G=(N, E)$.
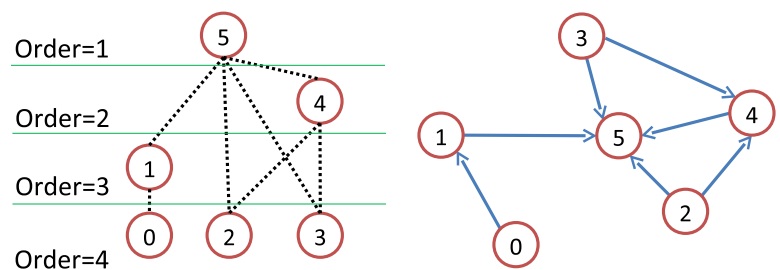
independent sets. Here dotted This step constructs the dilines are the edges in $E$. (Line rected edges from nodes 19 in Algorithm 2 in higher order to nodes in lower order (Line 10,17 in Algorithm 2]
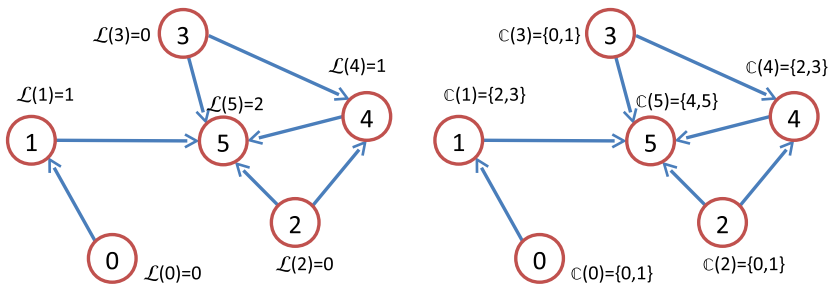

(d) Calculate $\mathcal{L}(i)$ to each node. (e) Calculate $\mathbb{C}(i)$ to each node (Line 19 in Algorithm 3) (Line 1023 in Algorithm 3).

Fig. 3. An example of the allocation algorithm for 6 access points and 6 channels. $(N=$ $\{0,1,2,3,4,5\}, C=\{0,1,2,3,4,5\})$

Definition 4 (Charging formula). For any player $i$ and any strategy profile $s=$ $\left(s_{i}, s_{-i}\right)$, the charge of player $i$ is

$$
\mathcal{P}_{i}(s) \triangleq \alpha\left(T_{i}\left(s_{i}^{*}, s_{-i}\right) / 2+T h\left(\left|s_{i}^{*} \backslash s_{i}\right|\right)+A\right),
$$

where

$$
A=\frac{\left|s_{i} \backslash s_{i}^{*}\right| T h^{2}\left(\left|s_{i}\right|\right)}{4\left(T_{i}\left(s_{i}^{*}, s_{-i}\right)+T h\left(\left|s_{i}^{*} \backslash s_{i}\right|\right)\right)} .
$$

For $\mathcal{P}_{i}(s)$, the first term $T_{i}\left(s_{i}^{*}, s_{-i}\right) / 2$ is an essential part of charge. The second term $T h\left(\left|s_{i}^{*} \backslash s_{i}\right|\right)$ and the third term $A$ together forms an external part of charge. This external part treats as a punishment, an additional charge. When the player obeys $s_{i}^{*}$, this part is zero, which means no penalty is imposed. However, when it does not obey $s_{i}^{*}$ and behave $s_{i}$, this part varies and increase higher than the additional utility obtained by $s_{i}$.

Then, we prove the strategy profile $s^{*}$ is a DSE.

Theorem 2. The channel allocation strategy profile $s^{*}$ computed by Algorithm 3 is a DSE under the charging scheme.

Proof. For any profile $s=\left(s_{i}, s_{-i}\right)$, the utility of player $i, u_{i}\left(s_{i}, s_{-i}\right)$ is 


$$
u_{i}\left(s_{i}, s_{-i}\right)=\alpha\left(T_{i}\left(s_{i}, s_{-i}\right)-\mathcal{P}_{i}(s)\right) .
$$

If $i$ chooses $s_{i}^{*}$ as its strategy,

$$
u_{i}\left(s_{i}^{*}, s_{-i}\right)=\alpha\left(T_{i}\left(s_{i}^{*}, s_{-i}\right) / 2\right) .
$$

Omitting the coefficient $\alpha$,

$$
\begin{aligned}
& 1 / \alpha\left(u_{i}\left(s_{i}^{*}, s_{-i}\right)-u_{i}\left(s_{i}, s_{-i}\right)\right) \\
= & T_{i}\left(s_{i}^{*}, s_{-i}\right)-T_{i}\left(s_{i}, s_{-i}\right)+T h\left(\left|s_{i}^{*} \backslash s_{i}\right|\right)+A \\
= & T_{i}\left(s_{i}^{*}, s_{-i}\right)-T_{i}\left(s_{i}, s_{-i}\right)+T h\left(\left|s_{i}^{*} \backslash s_{i}\right|\right)+\frac{\left|s_{i} \backslash s_{i}^{*}\right| T h^{2}\left(\left|s_{i}\right|\right)}{4\left(T_{i}\left(s_{i}^{*}, s_{-i}\right)+T h\left(\left|s_{i}^{*} \backslash s_{i}\right|\right)\right)} \\
\geq & -T_{i}\left(s_{i}, s_{-i}\right)+2 \sqrt{\frac{\left(T_{i}\left(s_{i}^{*}, s_{-i}\right)+T h\left(\left|s_{i}^{*} \backslash s_{i}\right|\right)\right) T h^{2}\left(\left|s_{i}\right|\right)}{4\left(T_{i}\left(s_{i}^{*}, s_{-i}\right)+T h\left(\left|s_{i}^{*} \backslash s_{i}\right|\right)\right)}} \\
= & -T_{i}\left(s_{i}, s_{-i}\right)+T h\left(\left|s_{i}\right|\right) \\
\geq & 0
\end{aligned}
$$

we have

$$
u_{i}\left(s_{i}^{*}, s_{-i}\right) \geq u_{i}\left(s_{i}, s_{-i}\right)
$$

Therefore we conclude that $s^{*}$ is DSE.

\section{Numerical Results}

We implement AMPLE and evaluate its performance using network simulations. The objective of our simulations is twofold. One is to test the performance of our channel allocation algorithm's outputs, which is the system-wide throughput. Since no proper existing works are comparable to our system, this evaluation compares the system-wide throughput achieved by anarchical NE and AMPLE's DSE. The other one is to verify that the system indeed converge to the DSE when AMPLE is used.

\subsection{Simulation Methodology}

In the simulation experiments, we use a basic CSMA/CA protocol with binary slotted exponential back-off as the MAC layer protocol. Following [22], the parameters used for the experiments are listed in Table 1

Metrics: We evaluate two quantitative values as metrics in this paper:

1. Utility: Utility is the difference between the player's valuation on throughput and charge for using the channels. This metric reflects the impacts of a player's behavior on its own.

2. System-wide throughput: It is the sum of all the players' throughputs. This metric is used to measure the effectiveness of our design on the performance of the channel allocation game. 
Table 1. Parameters used to obtain numerical results

\begin{tabular}{|l|l|}
\hline Packet Payload & 1450 bytes \\
PHY \&MAC Header & 50 bytes \\
ACK Packet Size & 30 bytes \\
\hline Minimum Contention Window & 32 \\
Number of Backoff Stages & 5 \\
\hline Original Channel Bit Rate & $1 \mathrm{Mbps}$ \\
Propagation Delay & $1 \mu \mathrm{s}$ \\
Slot Time & $50 \mu \mathrm{s}$ \\
SIFS & $28 \mu \mathrm{s}$ \\
DIFS & $128 \mu \mathrm{s}$ \\
ACK Timeout & $300 \mu \mathrm{s}$ \\
\hline
\end{tabular}

\subsection{Performance}

In this set of simulations, we evaluate the system-wide throughput of AMPLE and anarchical NE.

In the first simulation, we assume there are 20 access points and 12 channels. We set the degree of each point ranges from 3 to 5 and obeys a binomial distribution $(\sim \operatorname{Bin}(20,0.2))$, in which the average degree is 4 . The first simulation is repeated $10^{4}$ times. In each run, we generate a conflict graph, execute and record the system-wide throughputs of AMPLE and anarchical NE. Due to the limitation of space, we show the results of the first 50 runs in Figure 4. From Figure 4, we can observe that AMPLE gives relatively higher throughput than anarchical NE. Although anarchical NE gets higher throughput some times (almost twice every 25 runs), the average system-wide throughput of AMPLE is better than that of the anarchical NE. From this evaluation, the average ratio of the system-wide throughputs between AMPLE and anarchical NE is 1.1457, showing that AMPLE achieves an average of $15 \%$ higher throughput than that of anarchical NE.

In the second evaluation, we fix the number of access points at 20 , and vary the number of channels among 3,6,8, and 12. Other settings are the same as the first evaluation. In this evaluation, we repeat each simulation until the convergence level $10^{-4}$ is reached.

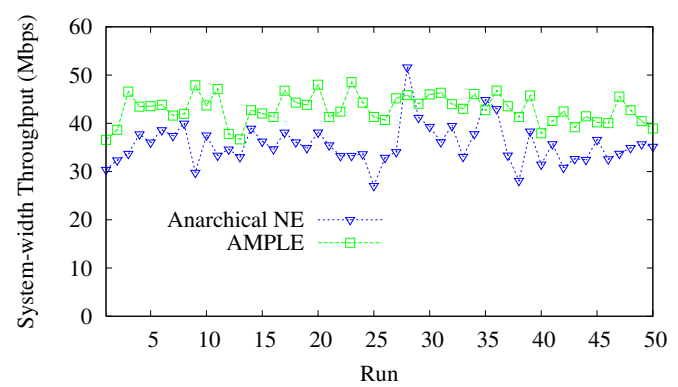

Fig. 4. The result of the first 50 runs of the simulation measuring the system-wide throughputs of AMPLE and anarchical NE. In each run, there are 20 access points and 12 channels. 


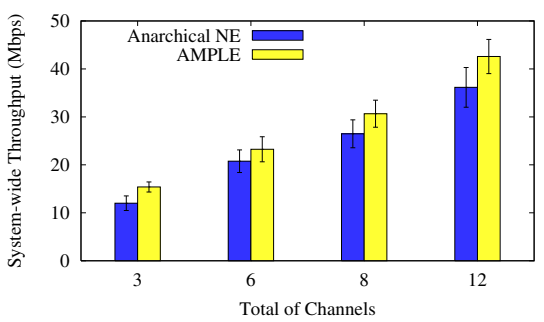

(a) The results of system-wide throughput achieved by AMPLE and anarchical NE when there are $3,6,8$, and 12 channels and 20 access points. The height of the bar show the average throughput, and the error-bar shows the standard deviation of the measured results.

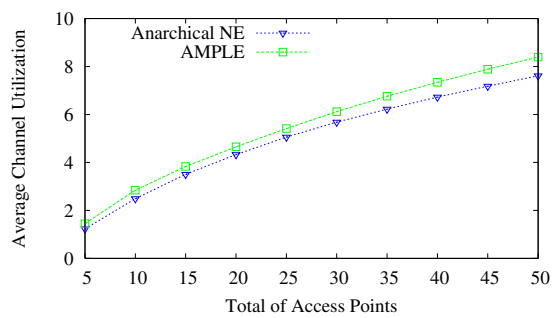

(c) The results of channel utilization achieved by AMPLE and anarchical NE when the total of access points varies from 5 to 50 . The number of channels is 12 .

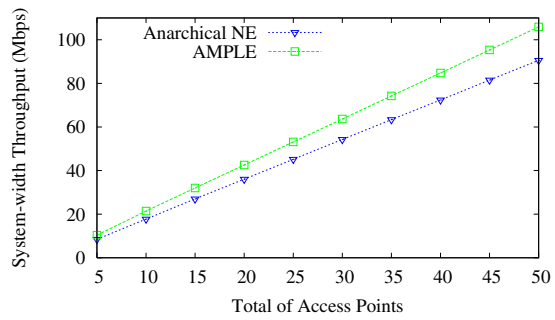

(b) The results of system-wide throughput achieved by AMPLE and anarchical NE when the total of access points varies from 5 to 50 . The number of channels is 12 .

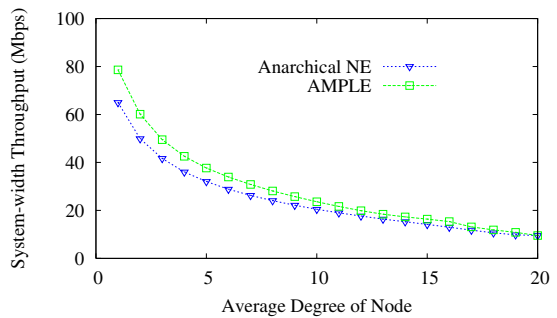

(d) The results of system-wide throughput achieved by AMPLE and anarchical NE when node degree varies from 1 to 20 .

Fig. 5. Experiment results for the first to the forth evaluations

Figure 5(a) illustrates the results. We can see that AMPLE always achieves higher system-wide throughput than anarchical NE does. At the same time, the standard deviations of AMPLE's results are also relatively smaller, which shows that the performance of AMPLE is more stable.

In the third evaluation, we vary the number of access points, while fixing the number of channels at 12 . We simulate the number of access points from 5 to 50 . Other settings are the same as the first simulation. In this evaluation, we also repeat each simulation until the convergence level $10^{-4}$ is reached.

Figure 5(b) shows that both the system-wide throughput of AMPLE and anarchical NE increase with the number of access points. However, AMPLE's throughput is larger than that of anarchical NE, and the gap between AMPLE and anarchical NE grows with the number of nodes.

We also record the average utilization of a channel in the third evaluation. Here, channel utilization means the average number of access points allocated to each channel. Figure 5(c) shows that the channel utilization of AMPLE is always higher than that of anarchical NE. 
In the forth evaluation, we vary the average degree of the access points from 1 to 20 , while the other settings are the same as the first simulation. Figure 5(d) shows that the system-wide throughput of both AMPLE and anarchical NE decreases when the network become more and more denser. However, AMPLE still always achieve better average system-wide throughput than anarchical NE.

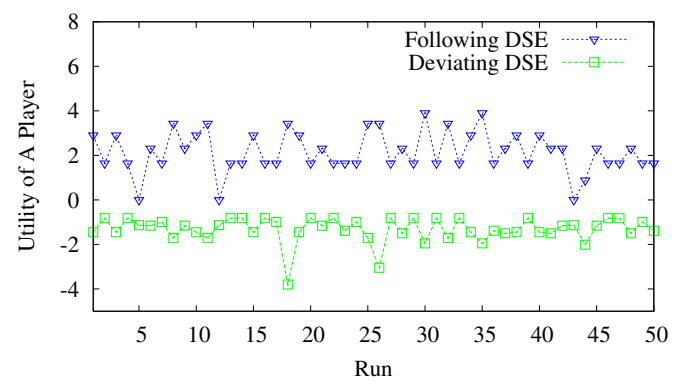

Fig. 6. The utility of a player when it follows or deviates from the channel allocation computed by AMPLE

\subsection{Truthfulness of AMPLE}

In this set of evaluations, we assume that $20 \%$ players are not following the channel allocation computed by AMPLE. We assume there are 12 channels, 20 players, and the average degree of the access points is 4 . In each run, we randomly pick 4 misbehaving access points and let them deviate from the channel allocation computed by AMPLE. Then we record the utility got by a fifth access point in 50 runs.

Figure 6 shows that the utility of a player when it follows or deviates from the channel allocation computed by AMPLE. We can observe that following the computed channel allocation is always no worse than that of deviating from it. Besides, the utility when following the scheme is always positive while the utility when deviating it is usually negative. So when AMPLE is used, the incentives for following the computed channel allocation is always guaranteed, no matter what the other players do. This result verifies that players cannot benefit by deviating from the channel allocation computed by AMPLE, when our charging scheme is used. Therefore, the convergence to the DSE is guaranteed on our charging scheme.

\section{Related Work}

In this section, we review the related work in this field. Felegyhazi et al. [9] first proposed a game model for the static multi-radio multi-channel allocation. Wu et al. [24] later put forward a mechanism to converge the multi-radio multi-channel allocation game to the strongly dominant strategy equilibrium (SDSE). They both only considered the problem in a single collision domain which is different from the scenario we consider in this paper. Recently, a number of strategy-proof auction-based spectrum allocation mechanisms (e.g., TRUST [30], SMALL [23], and VERITAS [29]) have been 
proposed to solve the problem in multiple collision domain. An important relevant work on channel allocation game is [12], in which the authors modeled it as a graph coloring problem and discussed the price of anarchical state under various topology conditions. However, none the the above work considers adaptive-width channels. A latest work by Wu et al. [22] discussed the allocation in adaptive-width channels. However, it is only valid in a single collision domain as well.

In wireless networks, the game theory is also applied to study problems such as media access. For example, MacKenzie et al. [14] studied the behaviors of selfish nodes in Aloha networks. Later, Cagalj et al. [5] and Konorski [13] utilized game-theoretic approaches to investigate the media access problem of selfish behavior in CSMA/CA networks. Nie and Comaniciu [16] proposed a game theoretic framework to study the behavior of cognitive radios for distributed adaptive spectrum allocation in cognitive radio networks. Some other relevant works on incentive-compatibility in wireless networks are [2, 3, 7, 8, 19, 21, 25, 28].

\section{Conclusion and Future Work}

In this paper, we proposed an approach for adaptive-width channel allocation in multihop, non-cooperative wireless networks. We first gave an algorithm to compute an efficient channel allocation, and then presented a charging scheme to guarantee that it is to the best interest of each player to follow the computed channel allocation. Evaluation results showed that our approach achieved good performance. As for future work, there can be several potential directions. One of the possible direction is to consider the case, in which the access points can be carefully to partially overlapping channels.

\section{References}

1. Second rep. and order and memorandum opinion and order, http://hraunfoss.fcc.gov/edocs_public/attachmatch/ FCC-08-260A1.pdf

2. Anderegg, L., Eidenbenz, S.: Ad hoc-VCG: a truthful and cost-efficient routing protocol for mobile ad hoc networks with selfish agents. In: Proceedings of the Ninth International Conference on Mobile Computing and Networking (MobiCom), San Diego, CA (September 2003)

3. Ben Salem, N., Buttyan, L., Hubaux, J.P., Jakobsson, M.: A charging and rewarding scheme for packet forwarding in multi-hop cellular networks. In: Proceedings of the Fourth ACM Symposium on Mobile Ad Hoc Networking and Computing (MobiHoc), Annapolis, MD (June 2003)

4. Bianchi, G.: Performance analysis of the IEEE 802.11 distributed coordination function. IEEE Journal on Selected Areas in Communications 18(3), 535-547 (2000)

5. Čagalj, M., Ganeriwal, S., Aad, I., Hubaux, J.-P.: On selfish behavior in CSMA/CA networks. In: Proceedings of 24th Annual IEEE Conference on Computer Communications (INFOCOM), Miami, FL (April 2005)

6. Chandra, R., Mahajan, R., Moscibroda, T., Raghavendra, R., Bahl, P.: A case for adapting channel width in wireless networks. In: Proceedings of ACM SIGCOMM 2008 Conference on Applications, Technologies, Architectures, and Protocols for Computer Communications, Seattle, USA (August 2008) 
7. Deek, L.B., Zhou, X., Almeroth, K.C., Zheng, H.: To preempt or not: Tackling bid and timebased cheating in online spectrum auctions. In: INFOCOM, pp. 2219-2227 (2011)

8. Eidenbenz, S., Resta, G., Santi, P.: Commit: A sender-centric truthful and energy-efficient routing protocol for ad hoc networks with selfish nodes. In: Proceedings of the 19th International Parallel and Distributed Processing Symposium (IPDPS), Denver, CO (April 2005)

9. Félegyházi, M., Čagalj, M., Bidokhti, S.S., Hubaux, J.-P.: Non-cooperative multi-radio channel allocation in wireless networks. In: Proceedings of 26th Annual IEEE Conference on Computer Communications (INFOCOM), Anchorage, AK (May 2007)

10. Fudenberg, D., Tirole, J.: Game Theory. MIT Press (1991)

11. Gummadi, R., Balakrishnan, H.: Wireless networks should spread spectrum based on demands. In: Proceedings of ACM Hotnets, Calgary, Canada (October 2008)

12. Halldórsson, M.M., Halpern, J.Y., Li, L.E., Mirrokni, V.S.: On spectrum sharing games. In: Proceedings of the 23rd Annual ACM SIGACT-SIGOPS Symposium on Principles of Distributed Computing (PODC), St. John's, Canada (July 2004)

13. Konorski, J.: Multiple Access in Ad-Hoc Wireless LANs with Noncooperative Stations. In: Gregori, E., Conti, M., Campbell, A.T., Omidyar, G., Zukerman, M. (eds.) NETWORKING 2002. LNCS, vol. 2345, pp. 1141-1146. Springer, Heidelberg (2002)

14. MacKenzie, A.B., Wicker, S.B.: Stability of multipacket slotted Aloha with selfish users and perfect information

15. Moscibroda, T., Chandra, R., Wu, Y., Sengupta, S., Bahl, P., Yuan, Y.: Load-aware spectrum distribution in wireless lans. In: Proceedings of the 16th International Conference on Network Protocols (ICNP) (October 2008)

16. Nie, N., Comaniciu, C.: Adaptive channel allocation spectrum etiquette for cognitive radio networks. In: Proceedings of the First IEEE International Symposium on New Frontiers in Dynamic Spectrum Access Networks (DySPAN), Baltimore Harbor, MD (November 2005)

17. Osborne, M.J., Rubenstein, A.: A Course in Game Theory. MIT Press (1994)

18. Rahul, H., Edalat, F., Sodini, D.K.C.: Frequency-aware rate adaptation and mac protocols. In: Proceedings of The Fourteenth International Conference on Mobile Computing and Networking (MobiCom), San Francisco, CA (September 2009)

19. Srinivasan, V., Nuggehalli, P., Chiasserini, C.-F., Rao, R.: Cooperation in wireless ad hoc networks

20. Wang, W., Eidenbez, S., Wang, Y., Li, X.-Y.: Ours-optimal unicast routing systems in noncooperative wireless networks. In: Proceedings of The Twelfth International Conference on Mobile Computing and Networking (MobiCom), Los Angeles (September 2006)

21. Wang, W., Li, X.-Y., Wang, Y.: Truthful multicast in selfish wireless networks. In: Proceedings of the Tenth International Conference on Mobile Computing and Networking (MobiCom), Philadelphia, PA (September 2004)

22. Wu, F., Singh, N., Vaidya, N., Chen, G.: On adaptive-width channel allocation in noncooperative, multi-radio wireless networks. In: Proceedings of 30th Annual IEEE Conference on Computer Communications (INFOCOM), Shanghai, China (April 2011)

23. Wu, F., Vaidya, N.: Small: A strategy-proof mechanism for radio spectrum allocation. University of Illinois at Urbana-Champaign, Tech. Rep. (2010)

24. Wu, F., Zhong, S., Qiao, C.: Globally optimal channel assignment for non-cooperative wireless networks. In: Proceedings of 27th Annual IEEE Conference on Computer Communications (INFOCOM), Phoenix, AZ (April 2008)

25. Xu, P., Xu, X., Tang, S., Li, X.-Y.: Truthful online spectrum allocation and auction in multichannel wireless networks. In: INFOCOM, pp. 26-30 (2011) 
26. Zhong, S., Li, L.E., Liu, Y.G., Yang, Y.R.: On designing incentive-compatible routing and forwarding protocols in wireless ad-hoc networks-an integrated approach using game theoretical and cryptographic techniques. In: Proceedings of The Eleventh International Conference on Mobile Computing and Networking (MobiCom), Cologne, Germany (September 2005)

27. Zhong, S., Chen, J., Yang, Y.R.: Sprite, a simple, cheat-proof, credit-based system for mobile ad-hoc networks

28. Zhong, S., Wu, F.: On designing collusion-resistant routing schemes for non-cooperative wireless ad hoc networks. In: Proceedings of The Thirteenth International Conference on Mobile Computing and Networking (MobiCom), Montreal, Canada (September 2007)

29. Zhou, X., Gandhi, S., Suri, S., Zheng, H.: ebay in the sky: Strategy-proof wireless spectrum auctions. In: Proceedings of The Fourteenth International Conference on Mobile Computing and Networking (MobiCom), San Francisco, CA (September 2008)

30. Zhou, X., Zheng, H.: Trust: A general framework for truthful double spectrum auctions. In: Proceedings of 28th Annual IEEE Conference on Computer Communications (INFOCOM), Rio de Janeiro, Brazil (April 2009) 